\title{
New possibility of retrospective EPR dosimetry
}

\author{
I. Vorona, S. Ishchenko, S. Okulov, T.T. Petrenko \\ Institute of Semiconductor Physics, pr. Nauky 45, Kyiv, 03028, Ukraine
}

\begin{abstract}
Tooth enamel plates irradiated by different types of radiation were studied by electron paramagnetic resonance imaging with local gradient of magnetic field. The dependence of radiation defect distributions on an irradiation type was found. A new procedure of retrospective electron paramagnetic resonancedosimetry determining the irradiation type was proposed.
\end{abstract}

Keywords: dosimetry, electron paramagnetic resonance, electron paramagnetic resonance imaging, tooth enamel

Paper received 18.09.99; revised manuscript received 07.12.99; accepted for publication 21.03.00.

\section{Introduction}

The study of the radiation influence on a living system and a human organism has become very important during last years. An irradiation dose and its power determine a result of such influence. Therefore, a dose evaluation is currently central. Nevertheless, immediate dose measurements during an irradiation process are often impossible, in particular, during accidents. The retrospective dosimetry on crystals of a biological origin (biocrystals) is very useful in such situations. The biocrystals are synthesized in living organisms and have little changes when time passes. Therefore, if structural defects were created in the biocrystals by radiation such defects remain constant for a long time and can be used as an irradiation retrospective detector. The retrospective electron paramagnetic resonance (EPR) dosimetry was applied to a variety of calcified tissues: bone, egg shells, mollusks, tooth enamel, tooth dentin, etc [1-6]. The stability of the main dosimetric signals in these materials is such that they may be used for EPR dating of archeological findings [7].

The tooth enamel is the most interesting biocrystal. It contains a mineral phase representing $94-98 \%$ of the total weight; water and organic matter form the rest. The inorganic component of enamel is largely composed by calcium apatites. The crystallites of hydroxyapatite are organized into small prisms plunged into an organic substance. Both prisms and crystallites reveal a certain ordering [8].

An ionizing irradiation of the tooth enamel induces paramagnetic centers responsible for intensive EPR spectrum near $\mathrm{g}=2$. The $\mathrm{CO}_{2}^{-}, \mathrm{CO}_{3}{ }^{3-}, \mathrm{CO}_{3}^{-}, \mathrm{CO}^{-}$radicals, $\mathrm{O}^{-}$ center and the so-called «background signal» with $g=2.0045, \Delta H_{p p}=0.64 \mathrm{mT}$ contribute to it [9]. The background signal is observed in the enamel samples before irradiation and dominates at irradiation doses up to $1 \mathrm{~Gy}$. The EPR signal intensity is a linear function of the irradiation dose in a wide dose range and is used in the retrospective dosimetry.

A characteristic feature of the retrospective EPR dosimetry procedure is the necessity of an additional laboratory irradiation to eliminate individual peculiarities of a tooth radiation sensitivity [10]. However, the EPR signal intensity was found to depend not only on an absorption dose but also on some radiation characteristics such as its type $(\beta, \gamma$, etc. $)$ and energy. Therefore, the determination of these parameters is important before application of the above procedure. Besides, there are problems as to differentiation of accidental ( $\gamma$-rays, as a 


\section{Vorona et al.: New possibility of retrospective EPR dosimetry}

rule) and medical (X-rays) irradiation. The procedure of a separate estimation of doses absorbed by front and back tooth parts has been applied up to now [3].

On the other hand, methods of the EPR imaging for obtaining distribution of paramagnetic defects along a sample are known [11-13]. The imaging methods appear to be useful in the retrospective EPR dosimetry because irradiation energy and type determine a radiation defect distribution along dosimeter. So some parameters of irradiation can be estimated from EPR images.

\section{Materials and methods}

Intact teeth with clinically sound enamel were used in this study. After removing dentin with diamond tools, four rectangular plates were cut off with the sizes approximately $4 \times 3 \times 1 \mathrm{~mm}^{3}$. Three of these plates were irradiated by electrons with $1.5 \mathrm{MeV}$ energies, $\gamma$ - and X-rays, respectively, the fourth one was irradiated by $1.5 \mathrm{MeV}$ energy electrons and $\gamma$-rays. The radiation was directed normally to one plate surface while other ones were protected against the irradiation. The absorption doses were estimated to be approximately 100-300 Gy at different samples. The fifth plate of the same size was received from molar enamel of a wild boar that had lived in the Chernobyl Zone.

EPR imaging measurements were carried out on «Radiopan» X-band EPR spectrometer equipped by ZZG1 imaging unit. The ZZG1 unit forms the additional magnetic field $B^{\prime}=G x$ directed along the main field $B$ so that a sample is located in the total field $B-G x$, where $x$ is the spatial coordinate along $B$ and $G$ denotes the magnetic field gradient. The so-called «local gradient of magnetic field» $(G=$ const $)$ was used in this study. In this case the EPR image is recorded by main field sweeping and described by

$$
T(B) \approx \int_{0}^{l} R(x) S(B-G x) d x,
$$

where $l$ is the sample length along the field direction, $R(x)$ denotes the spatial distribution of paramagnetic centers and $S(B)$ is the EPR lineshape function. The spatial distribution $R(x)$ is extracted from (1) by means of Fourier method or iterative algorithm.

Fourier method. The equation (1) can be written as:

$T=R * S$

where * is the convolution symbol.

Fourier method employs the convolution theorem. The Fourier transform of the convolution of two functions is equal to the product of their Fourier transforms. Therefore, after Fourier transform, equation (2) becomes:

$F(T)=F(R) F(S)$ and

$R(x)=F^{-1}\{F(T) / F(S)\}$.

The largest part of all known spectra can be decomposed into Lorentzian and/or Gaussian lineshape functions. Since Fourier transform of a Lorentzian or Gaussian diminished with increasing independent variable in the Fourier space $\omega$ the problem of division by zero occurs at large $\omega$. The simplest solution to overcome this problem is the division limitation to a window $f(\omega)$ :

$R(x)=F^{-1}\{F(T) f(\omega) / F(S)\}$.

To obtain the spatial distribution from EPR images a homemade program was used in this study. It allows applying the Gauss windows

$$
f(\omega)= \begin{cases}\exp \left\{-\frac{\omega^{2}}{\omega_{f}^{2}} \ln \frac{1}{a}\right\}, & \omega<\omega_{f} \\ 0, & \omega>\omega_{f}\end{cases}
$$

Parameter $\omega_{f}$ depends on signal/noise ratio and is defined from a spectrum

$F(\omega)=F(S) F^{*}(S)$

as a maximum value of $\omega$, where $F(\omega)$ values exceed the noise level.

Iterative algorithm. The equation (1) can be integrated with respect to magnetic field from zero to $B$ :

$$
\int_{0}^{B} T(t) d t \approx \int_{0}^{l} R(x) \int_{0}^{B} S(t-G x) d x d t
$$

The equation (8) can be written as:

$$
F(B) \approx \int_{0}^{l} R(x) I(B-G x) d x
$$

where $I(B)$ is EPR absorption line and

$$
F(B)=\int_{0}^{B} T(t) d t
$$

The equation (9) can be transformed as following:

$$
\begin{aligned}
& R(x)=F\left(G x+B_{0}\right) /\left(I\left(B_{0}\right) l\right)+ \\
& +R(x)-\int_{0}^{l} R(y) I\left(G(x-y)+B_{0}\right) d y
\end{aligned}
$$

On the base of equation (10) we can form the next iterative procedure which is solved numerically: 


\section{Vorona et al.: New possibility of retrospective EPR dosimetry}

$$
\begin{aligned}
& R_{n}+1(x)=F\left(G_{x}+B_{0}\right) /\left(I\left(B_{0}\right) l\right)+ \\
& +R_{n}(x)-\int_{0}^{l} R_{n}(y) I\left(G(x-y)+B_{0}\right) d y \\
& R_{0}(x)=F\left(G_{x}+B_{0}\right) /\left(I\left(B_{0}\right) l\right)
\end{aligned}
$$

Zero order approximation corresponds to the assumption that $F(B)$ is determined only by concentration of the centers which are located in the region where magnetic field is equal to resonance value.

It is evident that if $\lim _{n \rightarrow \infty} R_{n}(x)$ exists then it will obey the equation (1). Also it can be accurately proved that this limit exists and gives a usual onepeak EPR absorption.

\section{Results and discussion}

The sample was placed in a microwave cavity so that the direction of the field gradient was coincident with the direction of irradiation. As well known, the radiationinduced EPR spectrum of a tooth enamel plate depends on the direction of an external magnetic field. Therefore, EPR spectrum recorded in such sample geometry has been used as the EPR lineshape function in the calculations of spatial distributions. It is shown in Fig. 1.

The EPR images of the four enamel plates are presented in Fig. 2. The field gradient was equal to $2 \mathrm{~T} / \mathrm{m}$. It allowed to obtain the spatial resolution approximately $0.4 \mathrm{~mm}$. Corresponding distributions are presented on Fig. 3. They have been obtained by means of the Fourier transform and iterative algorithm that lead to the same distribution pattern. Fourier transform method gives successful results provided the Gauss windows with $a=0.03$ is used. Note that the blurring of a front edge of distributions is due to a window applying.

The calculated distributions reflect the change of a radiation defect concentration in plates along the direction of irradiation. It is well seen that different irradia-

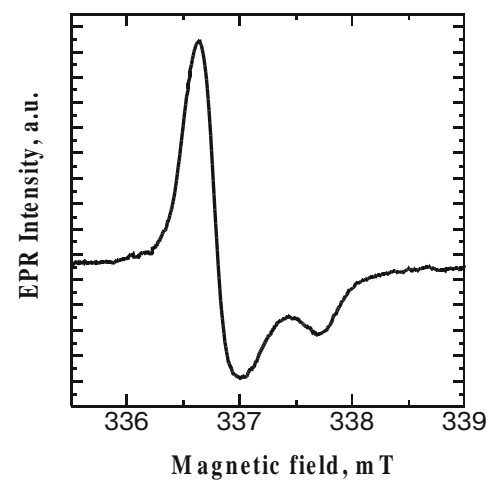

Fig. 1. EPR spectrum of irradiated tooth enamel plate. tion types result in different spatial distributions of paramagnetic defects. The $\gamma$-irradiation creates a homogeneous distribution of defects while a plate irradiated by electrons with $1.5 \mathrm{MeV}$ energy shows strong inhomogeneity in defect distribution (Fig. 2, a and c). The X-ray irradiation leads to an inhomogeneous distribution of defects with a detectable decay of their concentration along the sample (Fig. 2, b). The calculated distribution allows to separate visually the contributions of two irradiation types if their characteristics are distinctive (Fig. 2, d).

The fifth plate was studied to test the possibilities of the application of EPR imaging on real dosimetric objects. Recorded for different sample orientation, the EPR images showed homogeneous distribution similar to Fig. 2 , a. Thus, the samples cut out from tooth of the wild boar has been irradiated by $\gamma$-rays only. To determine the irradiation dose the standard procedure of the retrospective EPR dosimetry with additional g-irradiation was used. The dose was estimated to be approximately 16 Gy.

To eliminate the mistakes in the dose determination caused by different types irradiation the preparation of samples and the measurement procedure seem to be as following:

- getting a tooth;

- separating an enamel;

- cutting out a plate;

- measuring the EPR spectrum and EPR images;

- computing of a radiation defect distribution;

- separating the defects distribution on the components, each determined by one of the irradiation kinds;

- determining types of a sample irradiation;

- calculating the relative contribution of each irradiation type;

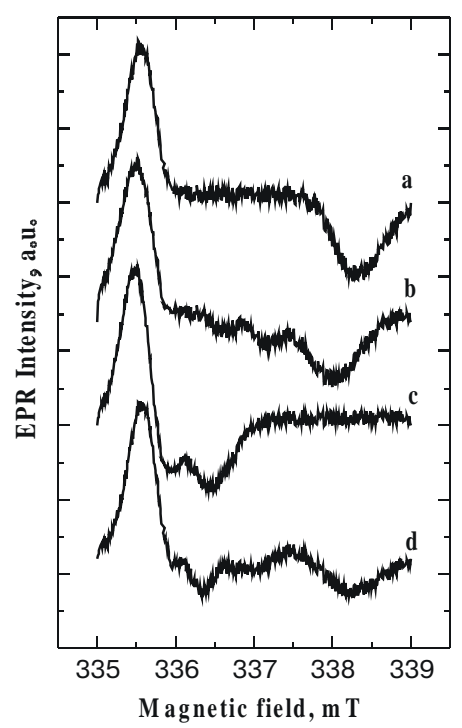

Fig. 2. EPR images recorded with $2 \mathrm{~T} / \mathrm{m}$ local gradient of magnetic field for tooth enamel plates irradiated by $g$-rays (a), Xrays (b), $1.5 \mathrm{MeV}$ electrons (c), $g$-rays and 1.5 MeV electrons (d). 


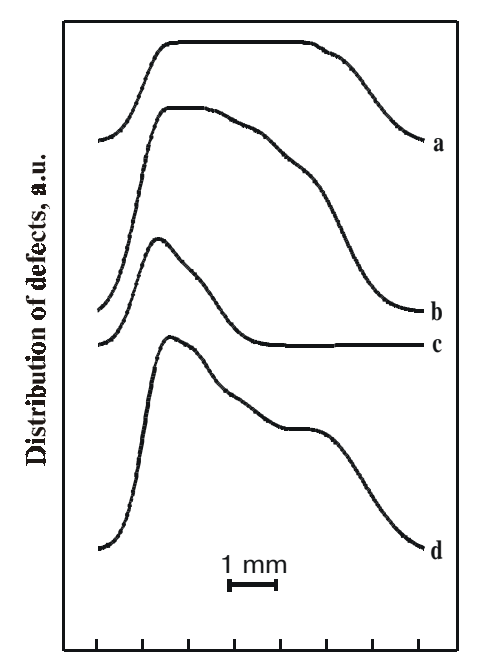

Fig. 3. The distributions of radiation defects obtained from EPR images in Fig. 2.

- dividing the sample into the parts according to the number of irradiation types;

- applying the standard procedure of the retrospective EPR dosimetry to each part of the samples with using the corresponding type of additional irradiation and taking into account the corresponding EPR component intensity;
- determining the total irradiation dose as the sum of doses received from different irradiation types

The use of EPR imaging in the retrospective dosimetry allows obtaining information about type and energy characteristics of sample irradiation. It permits to eliminate the dose determination mistakes caused by characteristics differences of accident and additional (laboratory) radiation.

\section{References}

1. D.F.Regulla, A.Wieser, H.Y.Goksu, Nucl. Tracks Radiat. Meas., 10, 825 (1984).

2. A.Kai, T.Miki, M.Ikeya, Quat. Sci. Rev., 7, 503 (1988).

3. T.Shimano, M.Iwasaki, C.Miyazawa, T.Miki, A.Kai, M.Ikeya, Appl. Radiat. Isot., 40, 1035(1989).

4. B.Pass, J.E.Aldrich, P.L.Scalion, Calcif. Tissue Int., 46, 166(1990).

5. A.B.Brik, S.S.Ishchenko, L.G.Rosenfeld, D.I.Zabolotnii, I.S.Zaritskaya, Med. Radiology, N1, p.25 (1993).

6. T.Nakajima, T.Otsuki, H.Hara, Appl. Radiat. Isot., 44, 91 (1993).

7. R.Grun, Appl. Radiat. Isot., 40, 1045 (1989).

8. E.Borovsky, Terapevticheskaya Stomatologiya, Meditsina, Moscow, 1988 (in Russian)

9. F.J.Callens, Nucleonika, 42, 565 (1997).

10. J.Tatsumi-Miyajima, Nuc. Instr. \& Meth., A257, 417(1987).

11. O.E.Yakimchenko, Ya.S.Lebedev, Sov. Chim. Phys., N4, 445(1983).

12. U.Ewert, T.-J.Herrling, Magnetic Resonance, 61, 11(1985).

13. G.R.Eaton, S.S.Eaton, K.Ohno, EPR Imaging and In Vivo $E P R$, CRC Press Inc., 1991. 